Revista Brasileira de Meteorologia, v.27, n.4, 435 - 446, 2012

\title{
SAZONALIDADE DA PRECIPITAÇÃO PARA A AMAZÔNIA USANDO O MODELO REGCM3: AVALIANDO APENAS A FORÇANTE DO ATLÂNTICO EQUATORIAL
}

\author{
WESLEY RODRIGUES SANTOS FERREIRA ${ }^{1}$, MARIA ISABEL VITORINO², EVERALDO \\ BARREIROS DE SOUZA², ALEXANDRE MELO CASSEB DO CARMO ${ }^{3}$
}

\author{
${ }^{1}$ Universidade Federal do Rio de Janeiro (UFRJ), Centro de Ciências Matemáticas e da Natureza (CCMN), \\ Instituto de Geociências (IGEO), Rio de Janeiro, RJ, Brasil \\ ${ }^{2}$ Universidade Federal do Pará, Instituto de Geociências, Faculdade de Meteorologia (UFPA/IG/FAMET), \\ Belém, PA, Brasil \\ ${ }^{3} \mathrm{UFPA} / \mathrm{IG}$, Faculdade de Oceanografia (FAOCE), Belém, PA, Brasil
}

rodrigueswesleysf@gmail.com, vitorino@ufpa.br, everaldo@ufpa.br, acasseb@ufpa.br

Recebido Maio de 2010 - Aceito Abril de 2012

\begin{abstract}
RESUMO
O presente estudo tenta trazer um novo ponto de vista sobre a importância do Oceano Atlântico Equatorial no padrão sazonal, durante o período de verão e de outono da região Amazônica. Desta maneira, contribui de uma forma diferente para os estudos na área de modelagem climática e variabilidade pluviométrica na região. Para isto, foi utilizado o modelo RegCM3, com esquema de convecção Grell, aplicando a técnica donwscaling e utilizando como condição inicial os dados de reanalise do NCEP/NCAR. Após a obtenção das simulações foi aplicado o método estatístico Viés para avaliar o quanto o modelo consegue reproduzir a precipitação sazonal. Em uma primeira análise, constata-se que o modelo é sensível a entrada de SF ou ZCAS, bem como, aproxima-se da dinâmica dos trópicos e extratropicos. Os resultados sugerem que a baixa resolução e a parametrização Grell são os principais fatores para a inadequação da qualidade das simulações. Apesar de o esquema Grell ser indicado para regiões com intensa convecção e fortes movimentos verticais, o mesmo necessita ser ajustado com as características físicas da região. Este estudo contribuiu para o aprimoramento dos modelos climáticos regionais para a região amazônica, considerando a contribuição dos processos oceano-atmosfera, durante o período de verão e outono do hemisfério sul.
\end{abstract}

Palavra chave: RegCM3/Grell, precipitação, ZCIT, efeitos locais.

ABSTRACT: SEASONALITY OF PRECIPITATION FOR THE AMAZON USING THE RegCM3 MODEL: EVALUATING ONLY THE FORCING OF EQUATORIAL ATLANTIC

The present study tries to bring a new perspective of the Equatorial Atlantic Ocean importance on seasonal pattern, during the summer and fall on the Amazon region. It contributes in a different way for studies in the area of climate modeling and variability of rainfall over the region. For this study, we used the model RegCM3 with Grell convection scheme, applying the donwscaling technique and using, as initial condition, NCEP/NCAR reanalysis data. After the simulations statistical method of the Bias was applied to evaluate how the accurately the model can reproduce the reanalysis seasonal rainfall. In a first analysis, it appears that the model is sensitive to FS or SAZC occurrences, as well as approaching the dynamics of the tropics and extratropics. The results suggest that the low resolution and the Grell parameterization are the main factors for inadequate quality of the simulations. Although the Grell scheme is suitable for areas with intense convection and strong vertical movements, an adjustment to the physical characteristics of the region is required. This study contributed to the improvement of regional climate models for the Amazon region, considering the contribution of ocean-atmosphere processes, during the summer and autumn in the southern hemisphere.

Keywords: RegCM3/Grell, precipitation, ITCZ, local effects 


\section{INTRODUÇÃO}

A previsão climática é importante para o planejamento sócio-econômico de um país ou de uma região, principalmente se a atividade desenvolvida for a agropecuária. Isto por que os recursos naturais e financeiros precisam ser bem aplicados, a fim de mitigar os danos e prejuízos causados pelo excesso ou falta de precipitação. Os sistemas meteorológicos precipitantes na Amazônia ocorrem em diferentes escalas de tempo-espaço, tais como: Zona de Convergência Intertropical (ZCIT), Zona de Convergência do Atlântico Sul (ZCAS), Vórtice Ciclônico de Altos Níveis (VCAN), Sistema Frontal (SF) e Linha de Instabilidade (LI) (Marengo; Hastenrath, 1993; Cohen et al., 1995; Figueroa e Nobre, 1990; Correia et al., 2007; Machado et al., 2004). Estes são modulados pelas variabilidades interanual e intrasazonal ( Vitorino et al., 2006; De Souza; Rocha, 2006; Ropelewski e Halpert, 1987; De Souza e Ambrizzi, 2003; De Souza et al.,2000).

Atualmente, os Modelos de Circulação Geral (MCG) da atmosfera que possuem domínio global apresentam diversas deficiências para previsão de clima, devido à baixa resolução em que trabalham (Menéndez et al., 2001; Misra et al., 2003; Nicolini et al., 2002; Quian et al., 2003). Todavia, apesar dos MCG serem capazes de representar os sistemas de grande escala (p. ex., ZCIT), responsáveis por grande parte da variabilidade da precipitação e da temperatura do ar, ainda não se mostram adequados para representar a dinâmica climática regional, tal como, o efeito de brisa marítima. De acordo com os autores supracitados, os MCG excluem completamente os processos de sub-grade, tais como, topografia, tipo de cobertura, distribuição de rios e lagos, curvatura da costa dos continentes e entre outros. Isto se torna uma limitação dos MCG, pois o clima é o resultado final da interação de vários processos físicos de diferentes escalas de espaço e tempo, em outras palavras, excluir tais efeitos regionais implica em eliminar uma parte importante que compõe o clima.

Os modelos regionais são capazes de capturar os efeitos da escala local, e assim, suprindo as limitações do MCG. Modelos de área limitada podem ser utilizados para estudos climáticos regionais baseando-se no conceito de donwscaling (p. ex. Hewitson e Crane, 1996; Wilks, 1999; Liang et al., 2006), que consiste em rodar um modelo regional "aninhado" a um modelo global, ou seja, os MCG fornecem as condições iniciais (contorno lateral e inferior) que contém informações dos padrões de circulação atmosférica da grande escala e o modelo regional tenta reproduzir os padrões de circulação de escala regional em alta resolução. Assim sendo, os Modelos Climáticos Regionais (MCR) mostram melhores resultados em suas simulações sazonais justamente por fazer este detalhamento mais amplo das características físicas do local.
Seth e Rojas (2003) realizaram simulações sazonais sobre a América do Sul e Oceano Atlântico adjacente, com um MCR aninhado a dados de reanalysis do National Center Environmental Prediction/National Center for Atmospheric Research (NCEP/NCAR). O MCR mostra-se capaz de identificar as principais diferenças de precipitação em dois anos extremos, como 1983 e 1985. Entretanto, o resultado mais interessante deste experimento é o indicativo de que nos trópicos as simulações dos MCR necessitam de domínios espaciais mais extensos do que em latitudes médias. Sugerindo, também, que a grande escala é a principal forçante para determinar a resposta da simulação de MCR sobre a América do Sul.

Cuadra e Rocha (2006) desenvolveram simulações de $50 \mathrm{~km}$ de resolução, com aplicação da técnica downscaling para representar a variabilidade da precipitação no Sudeste do Brasil, durante os verões de 1990 e 1998 . Os resultados sugerem que, os dados de um MCG alinhados, através de donwscaling, podem fornecer previsões climáticas mais detalhadas, porém com melhor qualidade.

Nobre et al. (2001) através das simulações realizadas para a estação chuvosa do nordeste do Brasil, janeiro a abril de 1999, com o Regional Spectral Model (RSM) do NCEP, em downscaling, com os dados do ECHAM3 do Atmospheric General Circulation Model (AGCM), verificaram que este "aninhamento" é capaz de gerar previsões chuvosas aproximadamente semelhantes com o observado e de melhor qualidade em comparação às previsões do modelo global. Onde, no primeiro momento, aplicou-se uma resolução de $80 \mathrm{~km}$ e em seguida, uma resolução de $20 \mathrm{~km}$ aninhada a "saídas" gerada pelo RSM-80 km. Da mesma forma, Sun et al. (2006) realizou algo semelhante, onde conseguiu representar alguns aspectos regionais do regime chuvoso para esta mesma região. Mas, no caso, se fez uso de um donwscaling do RSM com o ECHAM 4.5 AGCM, em uma resolução de $60 \mathrm{~km}$.

Recentemente, De Souza et al. (2009) avaliou o desempenho do RegCM3 para 26 períodos (1982/83 a 2007/08) entre verão e outono na Amazônia oriental, utilizando uma alta resolução $(30 \mathrm{~km})$. Um dos resultados satisfatórios é a habilidade que o modelo apresenta em representar o padrão sazonal na Amazônia oriental. Em anos anômalos desfavoráveis as chuvas estão associadas ao Dipolo do Atlântico positivo e El-Niño no Pacífico (De Souza e Ambrizzi, 2002), assim como, para os anos favoráveis as chuvas estão associadas a La Niña no Pacífico e Dipolo do Atlântico negativo (De Souza et al., 2004; De Souza e Ambrizzi, 2006). Apesar disto, o modelo apresenta erros sistemáticos na tentativa de representar os anos ditos normais. O esquema Grell, um dos esquemas de convecção presente no modelo, apresentou os melhores resultados nas simulações em comparação aos outros esquemas. Isto indica que o modelo é capaz de captar a variabilidade climática em anos anômalos, 
apesar do mesmo mostrar erros sistemáticos em se tratando de anos normais.

Dentro desta ótica, o objetivo central deste estudo é caracterizar o padrão sazonal da estação chuvosa para a região Amazônica, bem como, investigar a contribuição do Atlântico Equatorial no qualitativo da precipitação na Amazônia, utilizando para isso o modelo RegCM3. Para tanto, dados do NCEP/NCAR foram utilizados como condição inicial, através da técnica de donwscaling, e o esquema de convecção Grell (Grell, 1993) para representação de nuvens. Aplicou-se, ainda, a técnica do "viés" (De Souza et al., 2009.; Pal et al., 2000; Bergant et al., 2007 ) a fim de averiguar o quanto o modelo superestima ou subestima a precipitação.

\section{DADOS E METODOLOGIA}

\section{1 Área de estudo}

A região de estudo compreende a área de $16^{\circ} \mathrm{N}$ a $16^{\circ} \mathrm{S}$ e $80^{\circ}$ a $10^{\circ} \mathrm{W}$, que representa as regiões norte e nordeste do Brasil e Atlântico Equatorial. Este domínio foi selecionado por incluir os processos termodinâmicos relacionados com a atuação dos principais sistemas meteorológicos, como a ZCIT, responsáveis pelo maior quantitativo da precipitação local.

\subsection{Descrição do modelo RegCM3}

Neste trabalho, foi utilizado o RegCM3, desenvolvido e aprimorado pelo International Center for Theoretical Physics (ICTP). O RegCM3 é a terceira geração de um modelo climático regional originalmente desenvolvido no NCAR (Dickinson et al., 1989, Giorgi e Bates, 1989) - o RegCM1, o qual foi sistematicamente aprimorado por Giorgi et al. (1993a, 1993b) - RegCM2, Giorgi e Mearns, 1999 - o RegCM2.5. O RegCM3 é um modelo de área limitada, com grade horizontal de Arakawa-Lamb B, em coordenada vertical sigma de equações para um fluído compressível e hidrostático (Pal et al., 2007). Para resolver os processos de interação da superfície, tipo de solo e vegetação com a atmosfera, o RegCM3 usa o esquema de superfície Biosphere-Atmosphere Transfer Scheme versão 1e (BATS1e) (Dickinson et al., 1993), descrito como um pacote de superfície destinado a caracterizar o tipo de vegetação, a interatividade da umidade do solo e as trocas superfícieatmosfera de momento, energia e vapor d'água. Para representar o balanço de energia na atmosfera, o RegCM3 utiliza o esquema de transferência de radiação do NCAR Community Climate Model 3, descrito por Kiehl et al. (1998). Resumidamente, este esquema considera as componentes da energia solar, incluindo o efeito do $\mathrm{O} 3, \mathrm{H} 2 \mathrm{O}, \mathrm{CO} 2$ e $\mathrm{O} 2$, que segue a aproximação de $\delta$-Eddington, em que a radiação da nuvem depende de três parametrizações: a cobertura da nuvem fracionária, conteúdo de água liquida da nuvem e o efeito do raio da gotícula da nuvem. O espalhamento e absorção das nuvens seguem as parametrizações de Slingo (1989). O esquema do Community Climate Model (CCM) versão 3, mantém a mesma estrutura como a do CCM2, mas incluindo novos recursos, tais como: o efeito adicional dos gases do efeito estufa (NO2, CH4, CFCs), aerossóis atmosféricos e gelo das nuvens.

\subsubsection{Esquema de convecção (GRELL)}

A convecção é um processo físico natural bastante peculiar e instável. Isso ocorre por este processo apresentar uma ampla variação de espaço-tempo. Tipicamente, em regiões tropicais, as correntes ascendentes podem variar de 0,2 a $2 \mathrm{~km}$ ou mais (Molion, 1987; Arkin, 1987), perdurando de segundos a horas, ou seja, a formação de nuvem na região dos trópicos é fortemente dependente da convecção, principalmente quando se trata de nuvens precipitantes. Mas, apesar dos avanços na modelagem atmosférica, a representação explicita da convecção de nuvem ainda é um desafio para a comunidade científica, uma vez que, a principio, a resolução em que o modelo trabalha é insuficiente para representar um processo de tão alta resolução tempo-espaço. Portanto, estudiosos formularam um método em que este processo fosse implicitamente representado dentro do modelo, ou seja, os esquemas de convecção.

A principal característica do esquema de convecção Grell é considerar dois estados de circulação da nuvem, a saber, o umedecimento das correntes ascendentes e o aquecimento das correntes descendentes (Steiner et al., 2009, De Souza et al., 2009, Segele et al., 2009), de forma que o contato com o meio ambiente ocorre indiretamente, exceto no topo e na base da circulação. O fluxo de massa é constante com a altura e o arrasto ocorre junto à borda das nuvens. Os níveis originários do movimento ascendente e descendente são dados, respectivamente, pelos níveis de energia estática úmida máxima e mínima. O esquema Grell é ativado quando uma parcela de ar se eleva atingindo a convecção úmida. Por ser um esquema simplista, se torna necessário utilizar algum tipo de fechamento para completá-lo, de modo que, o fechamento calcula a quantidade de convecção que ocorre e o esquema de convecção Grell estima as propriedades desta convecção. Neste trabalho, utiliza-se o fechamento Fritsch-Chappell, no qual, a energia de flutuablidade acontece em uma escala de tempo de 30 minutos (Pal et al., 2007).

\subsection{Configuração das simulações}

Para a região da Amazônia, foram usados os dados de precipitação do Climate Prediction Center (CPC) pertencente 
ao NCEP. O CPC disponibiliza a base de dados de precipitação diária para o Brasil, a qual é gerada, através da interpolação espacial dos dados observados das estações meteorológicas de superfície e Plataformas de Coletas de Dados (PCD), reportada na codificação SYNOP das 1200 UTC. Essas estações são derivadas da rede observacional do Instituído Nacional de Meteorologia (INMET), Centro de Previsão de Tempo Estudos Climáticos (CPTEC), Agência Nacional Energia Elétrica (ANEEL), Agência Nacional de Águas (ANA) e diversos centros estaduais de meteorologia. $\mathrm{Na}$ geração desta base de dados, se fez uso de um controle de qualidade e métodos de interpolação espacial descritos detalhadamente em Silva et al. (2007). Estes dados são utilizados como referência para avaliação de o quanto o modelo é capaz de reproduzir o padrão de chuva observada. Para a avaliação do padrão oceânico do Atlântico Equatorial, são utilizados os dados de anomalia de Temperatura da Superficial do Mar (TSM) do NCEP/NCAR.

Em MCR, a "condição inicial”, é a junção dos dados ditos de "contorno lateral" e "contorno inferior". Entendido como o ponto de partida, de entrada dos dados (input), em que o modelo inicia sua "rodada" ou simulação. Neste trabalho, os dados do NCEP/NCAR (Kalnay et al., 1996) foram definidos como condição inicial, trazendo informações do clima observado em grande escala. Dessa forma, o RegCM3 tentará reproduzir este clima observado na escala regional. Em outras palavras, tentará reproduzir o efeito "feedback" em relação a grande escala, sendo que, durante toda a "rodada" o RegCM3 será "alimentado" continuamente com estes dados. Portanto, para o contorno lateral foram usados dados de seis em 6 horas das componentes zonal e meridional do vento, velocidade vertical, temperatura do ar, altura geopotencial, umidade relativa e pressão ao nível médio do mar. Estes dados foram gerados através de um consistente sistema de assimilação de dados empregados no MCG do NCEP e com resolução T62L28. Para contorno inferior, foram empregados dados de TSM semanal em grade regular global e resolução horizontal de $1.0^{\circ}$ latitude $\times$ $1.0^{\circ}$ longitude (Reynolds et al., 2002), os quais foram adquiridos junto ao $\mathrm{CPC}$ NCEP.

A Figura 1 ilustra o sistema de modelagem do RegCM3 instalado no Laboratório Rede Estadual de Previsão Climática e Hidrológica (RPCH) da Faculdade de Meteorologia da Universidade Federal do Pará (UFPa). O modelo RegCM3 usa o estado-da-arte em modelagem numérica, com processamento computacional em paralelo usando o compilador FORTRAN da Intel e o paralelizador MPI, que funciona em ambiente Linux OpenSUSE 10.0. Essa estrutura se encontra instalada num Cluster de Workstation que totaliza 48 processadores, sendo a máquina principal (máster) uma máquina HP com dois processadores Intel Xeon Quad Core, com 8GB de memória e HD com 2.5 TB (disco rígido) e os outros cinco nodos são as máquinas HP cada uma contendo dois processadores Intel Xeon Quad Core, com seis GB de memória e 1TB de HD. A etapa de pré-processamento se inicia no Terrain, onde é feita a escolha do domínio (número de pontos em $\mathrm{x}, \mathrm{y}, \mathrm{z}$ ), resolução espacial (em km), a projeção (mercator normal, etc), dados de entrada das condições de contorno inferior (TSM semanal, etc) e lateral (reanálise NNRP1, etc), tempo de integração e etc. Após a definição do Terrain, é processado o ICBC (Initial and Lateral Boundary Condition), no qual, são gerados os arquivos da condição de contorno inferior e lateral para o domínio escolhido. Em seguida, tem a etapa do Modelo. Esta etapa é realizada a rodada computacional que gera vários arquivos, os quais são posteriormente pós-processados.

Para este trabalho, foi adotada a seguinte configuração das simulações regionais: as rodadas foram realizadas usando a reanálise NCEP/NCAR (NNRP1), como contorno lateral atualizada a cada 6 horas, e a TSM semanal foi considerada como contorno inferior. A dimensão da grade para a Amazônia e Oceano Atlântico Equatorial, possui 96 pontos em longitude (x), 48 em latitude (y), com 18 níveis verticais ( $\mathrm{z}$ ) em coordenada sigma e espaçamento de grade de $80 \mathrm{~km}$, com a grade centrada em $1^{\circ} \mathrm{S}$ e $45^{\circ} \mathrm{W}$ e usando projeção mercator normal. Foram realizadas 5 simulações sazonais usando o esquema Grell com fechamento Fritsch-Chappell para a construção de um ensemble, sendo que todas as datas de início das simulações em 2008 foram: 27 de outubro para primeira previsão (PREV1), 03 de novembro - PREV2, 10 de novembro - PREV3, 17 de novembro - PREV4 e 24 de novembro - PREV5 e a data final das simulações foi em 31 de maio de 2009. Analisaram-se

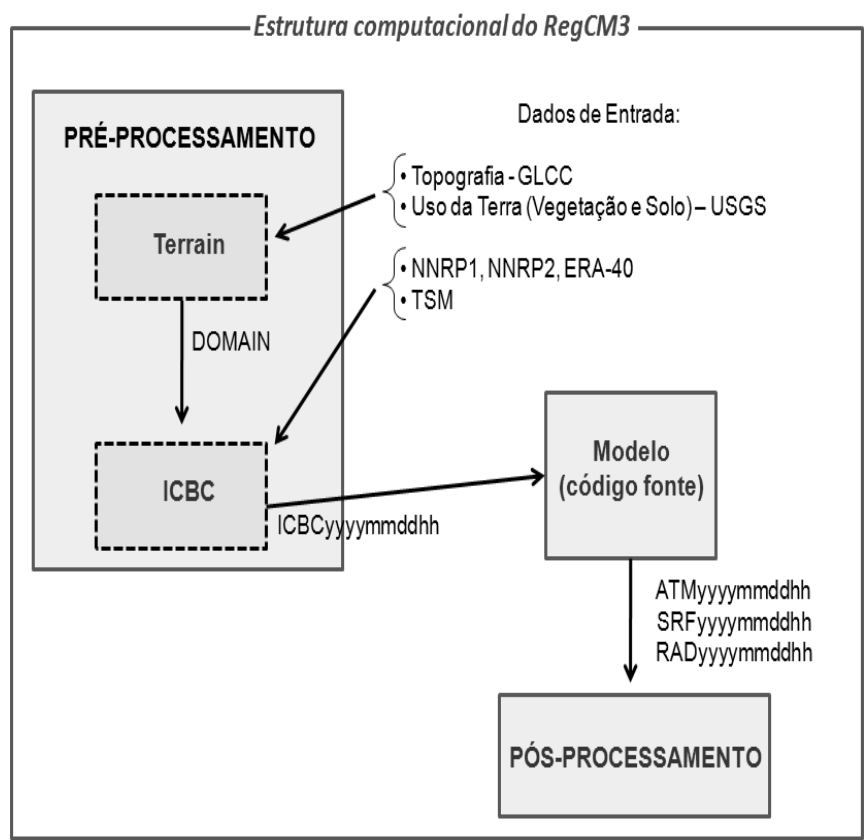

Figura 1 - Estrutura computacional do RegCM3. 
somente as saídas do período de 1 de dezembro de 2008 até 31 de maio de 2009, onde o período anterior é considerado como o tempo de "spi-up". Portanto, neste trabalho foram analisadas as simulações do período de verão (Dezembro a Fevereiro) e outono (Março a Maio) de 2008/2009.

\subsection{Métodos estatísticos de avaliação}

Em geral, a análise objetiva de comparação entre o observado e o simulado, é feita através do cálculo do viés para cada ponto de grade. O viés ou bias, como encontrado freqüentemente na literatura, é uma metodologia estatística utilizada para expressar o erro sistemático ou tendenciosidade no prognóstico de determinada variável (Kiehl et, al,. 1998, Lopes, 2009). Este método mede a correspondência entre a previsão média e o valor médio observado de um determinado parâmetro (Wilks, 2006). O viés negativo está indicando que o modelo tende a subestimar o determinado parâmetro simulado, quanto mais negativo, mais o modelo tende ao erro de subestimativa. E quanto mais o viés for positivo, mais o modelo tende a superestimar o parâmetro. Já quando o viés se aproximar do zero, o parâmetro está sendo mais bem representado, indicando que o modelo se aproxima do valor real.

Vies $=s-o$

Onde $s$ representa as simulações e $o$ representa a observação.

Na tentativa de se prolongar a previsão e a previsibilidade dos modelos dinâmicos, surgiu a idéia de se utilizar a técnica de ensemble (Leung et al., 2004; Buizza et al., 1999; Molteni et al., 1996). Um ensemble consiste em fazer várias rodadas de modelo com diferentes condições iniciais e, posteriormente, fazer a média desse conjunto (ensemble) de previsões. Considerando que cada rodada (membro) possa gerar condições futuras diferentes, se espera então, que o resultado do ensemble seja mais satisfatório que a previsão de um único membro, pois, teoricamente, um conjunto eliminará erros e/ou ruídos de um único membro.

\section{RESULTADOS}

\subsection{Padrões dinâmicos sazonais}

Nesta sessão, serão apresentados os padrões dinâmicos da circulação atmosférica para os períodos sazonais de DJF e MAM (2008/09). Estes, serão mostrados a partir dos escoamentos em $850 \mathrm{hPa}$ e $200 \mathrm{hPa}$ para os campos observados e a simulados pelo RegCM3/Grell, considerando a média dos 5 membros (ENSEMBLE).

As observações de verão e outono mostram que os ventos zonais, em superfície, estão mais intensos sobre o oceano Atlântico Equatorial e norte da América do Sul (AS), ou seja, englobando os setores norte/noroeste do estado do Pará e Amazonas e os setores leste/nordeste de Roraima e Amapá (Figura 2). Por outro, os ventos estão menos intenso no centrosul das regiões norte e nordeste. Em relação às simulações, o modelo RegCM3/Grell mostra semelhantes direções e intensidades para as variações espaciais e temporais, exceto com relação ao oceano próximo a costa norte da América do Sul. Nesta, o modelo coloca uma região de difluência do escoamento em superfície que pode estar associada com movimentos subsidentes, ou seja, ausência de nebulosidade (Figura 2).

A Figura 3 mostra que a circulação atmosférica de altos níveis está próximo ao padrão da climatologia de verão na AS, com a presença típica da Alta da Bolívia $(\mathrm{AB})$ e o cavado do nordeste $(\mathrm{CN})$, se estendendo para o litoral norte da AS. Na estação de outono, ainda se observa uma circulação de alta pressão deslocada para o norte, porém, mais zonal. Além disso, pode ser notado que no oceano Atlântico há a presença de dois centros de alta pressão que podem estar respondendo por centros de baixa pressão em superfície. Com relação às simulações o período de verão foi bem representado pelo modelo, no entanto, ao invés de reproduzir o cavado do nordeste, foi mostrado um Vórtice Ciclônico de Altos Níveis (VCAN). No outono, as circulações, em geral, foram reproduzidas como no observado, mas os centros das altas pressões foram deslocados para oeste (com relação à $\mathrm{AB}$ ) e para leste (relacionada às altas do oceano Atlântico).

Quando analisado simultaneamente as simulações em baixos e altos níveis no verão, onde se encontra o VCAN, é observado um ponto de divergência em $850 \mathrm{hPa}$ (Figura 2), indicando que o VCAN, alocado pelo modelo, é tão profundo ao ponto de inverter os ventos em baixos níveis contra o escoamento de leste. Isto mostra que, no outono e na mesma região do $\mathrm{VCAN}$, o RegCM3/Grell, apresenta resposta semelhante ao verão em superfície. Mas na alta atmosfera, o escoamento parece não estar relacionado com o comportamento dos ventos em superfície (Figuras 2 e 3). Estas diferenças podem estar relacionadas com a resolução espacial da grade e o esquema Grell, juntamente com a ausência da contribuição do oceano Pacifico Equatorial. Isto porque De Souza et al. (2009) mostraram, com resolução mais alta e inclusão de ambos oceanos, resultados mais expressivos com relação às observações.

\subsection{Precipitação sazonal}

Nesta sessão, discute-se o padrão da anomalia mensal da TSM (aTSM) sobre o Atlântico Equatorial (Figura 4), a fim de caracterizar as condições oceano-atmosfera durante o período de estudo. Além de, mostrar a distribuição espacial da precipitação 

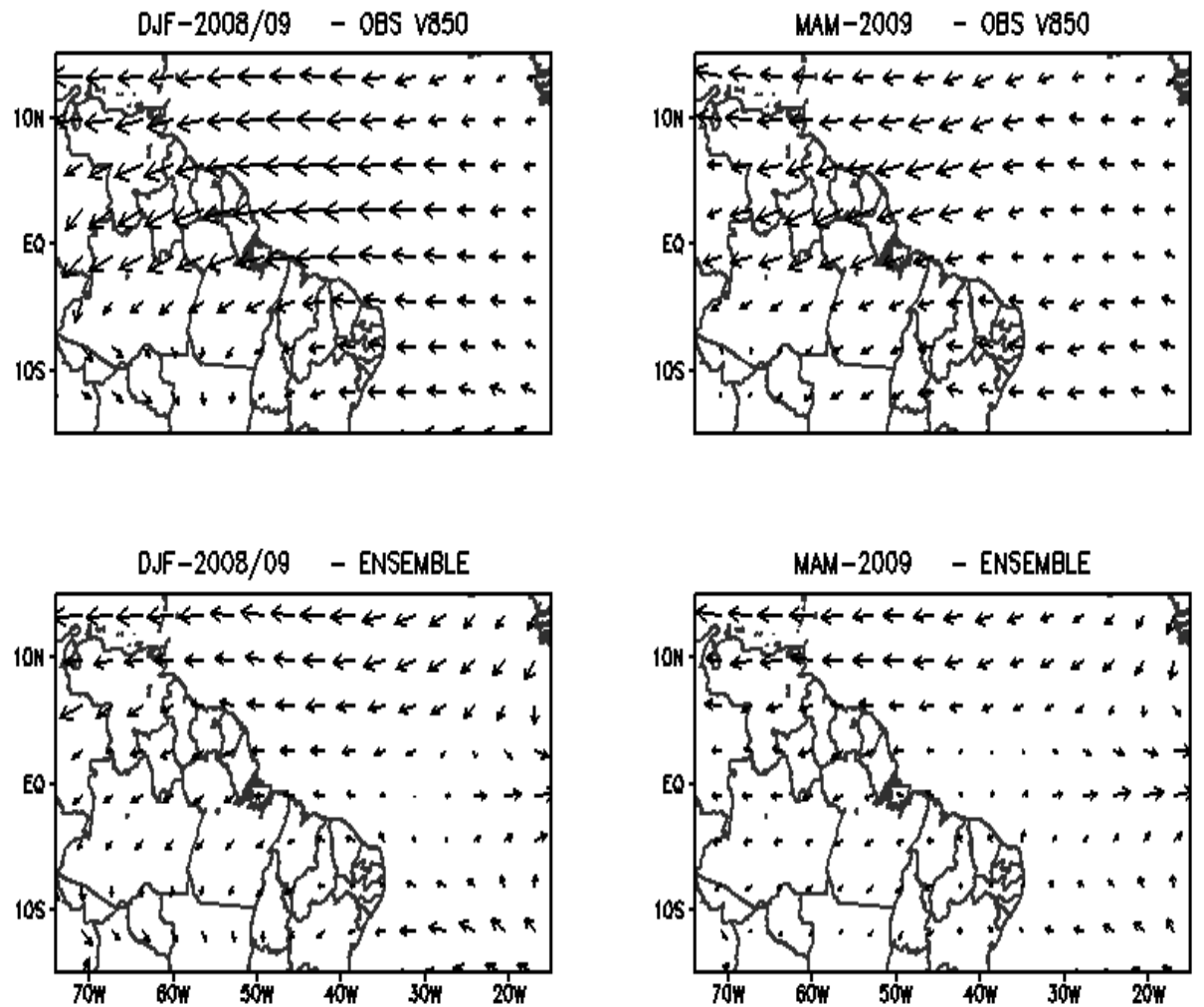

Figura 2 - Vento (m/s) em 850 hPa observado (OBS) e simulado (ENSEMBLE) pelo RegCM3/Grell para DJF-2008/09 (painel á esquerda) e MAM-2009 (painel á direita).
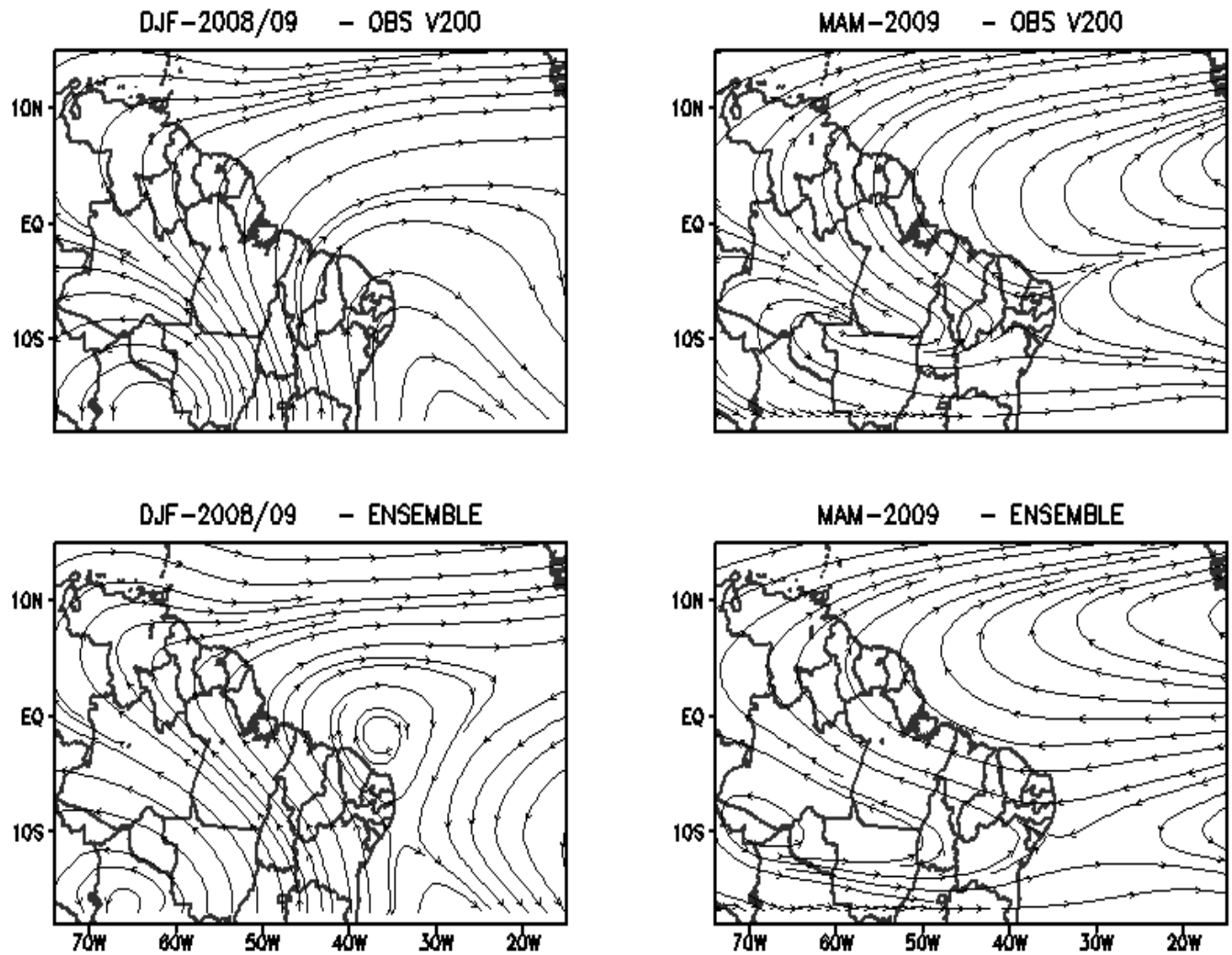

Figura 3 - Vento (m/s) em 200 hPa observado (OBS) e simulado (ENSEMBLE) pelo RegCM3/Grell para DJF-2008/09 (painel á esquerda) e MAM-2009 (painel á direita). 
sazonal observada de DJF e MAM (2008/09) e a precipitação sazonal simulada pelo RegCM3/Grell, considerando a média de cinco membros de previsão (ENSEMBLE) (Figura 5).

A aTSM do Atlântico Equatorial (Figura 4) se mostra positiva a leste e norte da $\mathrm{AS}$, se estendendo a sudeste da área de estudo e desintensificando até fevereiro. No entanto, ela volta a se intensificar em março, reduzindo em abril e retomando a intensidade em maio. A aTSM negativa se expressa com menor extensão espacial do que a aTSM positiva. Porém, a mesma surge em janeiro no extremo leste do Atlântico norte Equatorial, se intensificando entre os meses de fevereiro a abril. Apesar do Atlântico Equatorial não apresentar claramente um padrão de Dipolo negativo, semelhante ao que é descrito por Moura e Shukla (1981). Mas o aquecimento anômalo na bacia sul do Atlântico contribui para a atuação da ZCIT abaixo da linha do equador, dessa forma favorece a ocorrência de maiores volumes de chuvas na região norte e nordeste do Brasil. O fato do Pacifico Equatorial estar com aTSM dentro da normalidade e que estudos anteriores sugerem conexão via célula de Walker entre o Pacífico e o Atlântico, foi observado que durante episódios de ENOS, as aTSM do Atlântico podem estar associadas com a Oscilação do Ártico, através do centro de alta pressão do atlântico norte.

Durante o verão (Figura 5), foi observado que os maiores valores de precipitação ocorreram no nordeste da Amazônia, que inclui, principalmente, o nordeste do estado do Pará. A precipitação acumulada esteve acima de $300 \mathrm{~mm}$, com máximos acima de $400 \mathrm{~mm}$ sobre Belém e o litoral da capital. Estes máximos de chuva podem estar associados à atuação de sistemas frontais, de ZCAS, de LI e de aglomerados convectivos, devido aos efeitos locais (Cohen et al., 1995; Kousky e Cavalcanti,

\section{Dez/08}
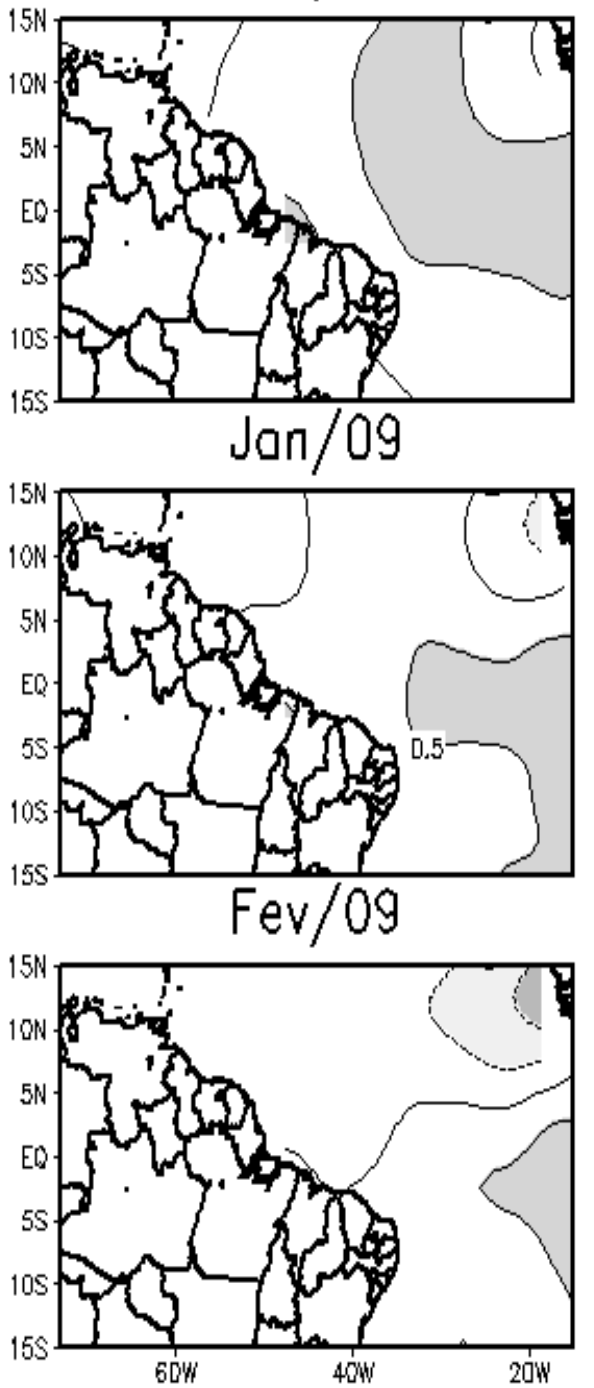

Mar/09
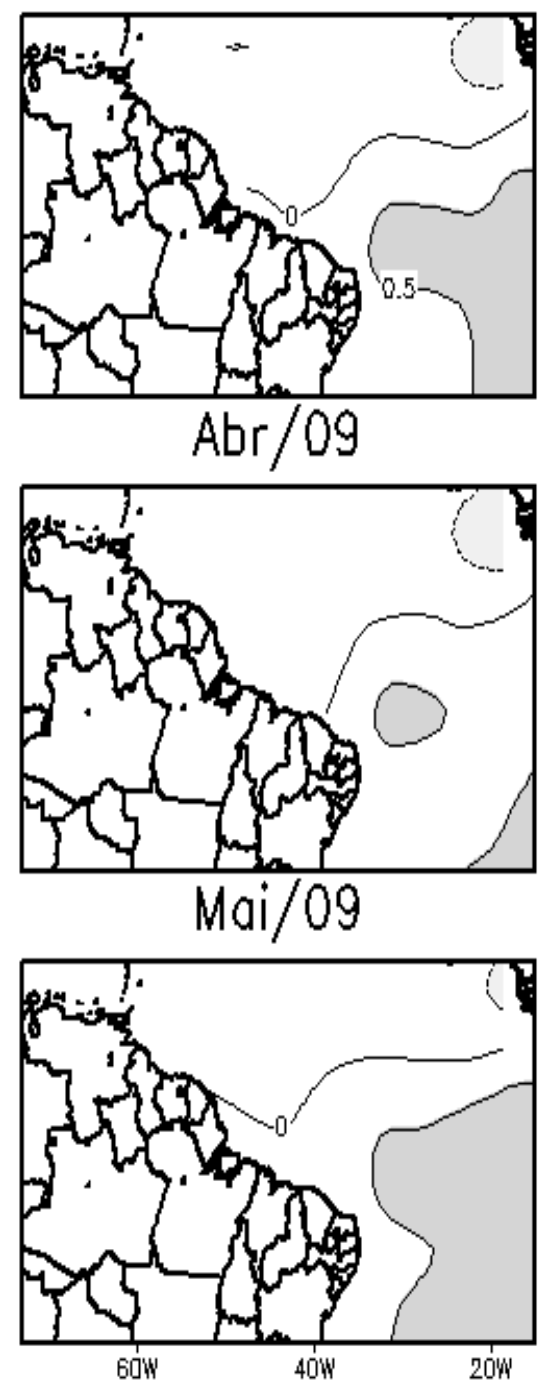

2.5

2

1.5

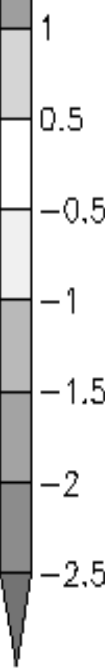

Figura 4 -Anomalia mensal da Temperatura Superficial do Mar (aTSM) sobre o Oceano Atlântico Equatorial entre dezembro de 2008 a maio de 2009 (linha contínua - positivo e linha tracejada - negativo). 

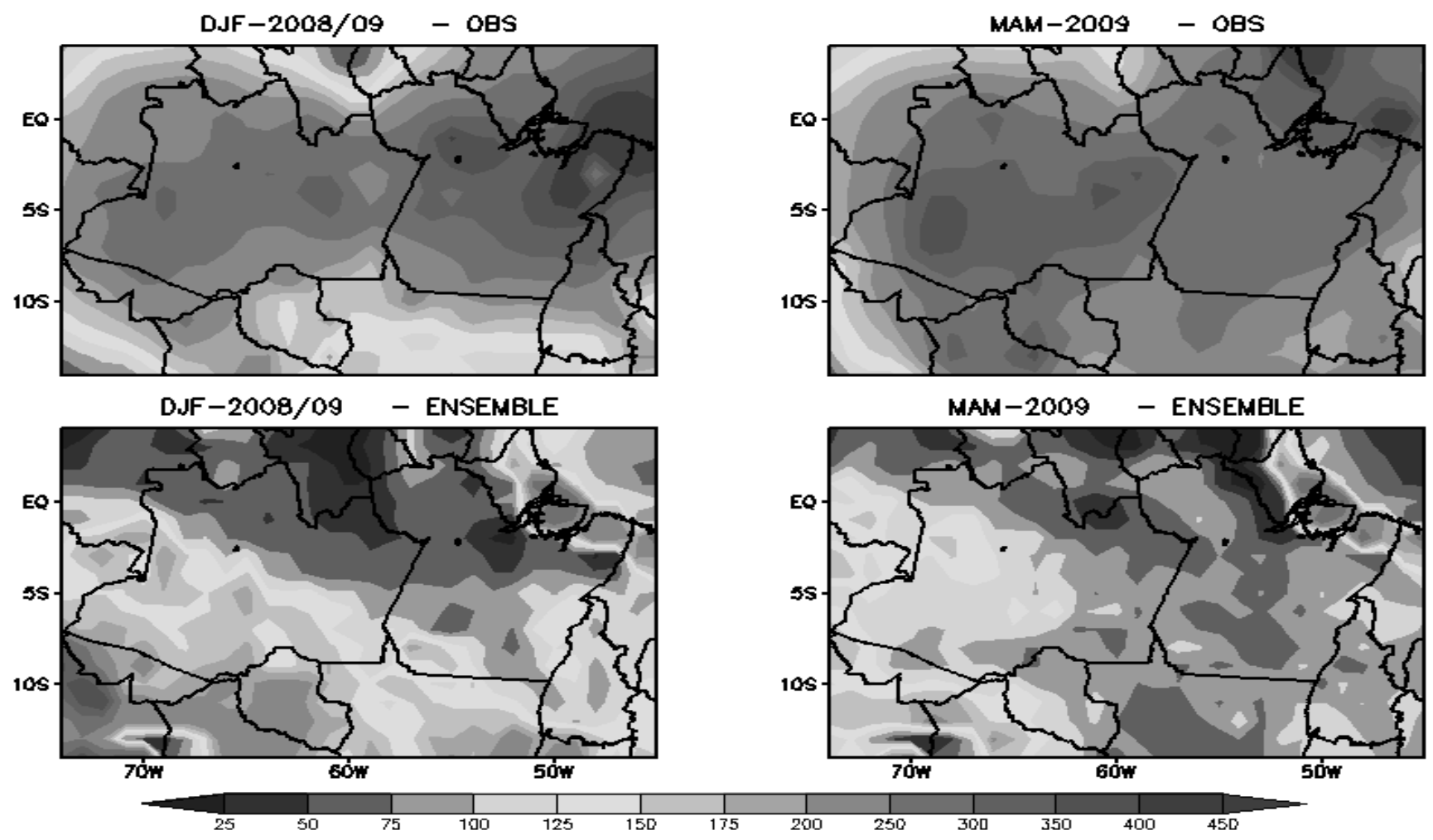

Figura 5 - Precipitação (mm/mês) sazonal para DJF-2008/09 (painel á esquerda) e MAM-2009 (painel á direita) sobre a Amazônia, com base nos dados observados e simulados pelo RegCM3/Grell para a média dos 5 membros (ENSEMBLE).

1988; Carvalho et al., 2002). Diferente do verão, o outono apresenta máximos de precipitação em grande parte do estado do Amazonas, litoral do Pará e Amapá. Nesta época, a ZCIT se encontra mais ao sul do equador, devido a influência das aTSM positiva do Atlântico Equatorial. Além disso, os efeitos locais da região, devido a cobertura vegetal e a atuação dos SF e ZCAS, podem responder por tal padrão espacial de precipitação sazonal.

De modo geral, o RegCM3/Grell, apesar de sua baixa resolução, mostrou habilidades em representar a chuva local causada pelas circulações de mesoescala nas proximidades do litoral do Pará, durante o verão e outono. Isto concorda com estudos observacionais de Linhas de Instabilidades que se formam nesta área (Cohen et al., 1995). Devido o modelo ser projetado para captar efeitos locais, é natural que o mesmo seja suscetível ao efeito de brisa já que se trata de um efeito local. Por outro lado, a sensibilidade que o esquema Grell apresenta, em relação ás correntes verticais, contribuem para este resultado uma vez que o tempo e o clima, na faixa costeira, são dependentes destes movimentos verticais para sua configuração (Pal et al., 2000; Pal et al., 2007). As configurações espaciais relacionadas à atuação dos sistemas sinóticos, como SF e ZCAS, foram representadas de modo incipiente, principalmente, durante o outono. No verão, ainda se pode perceber que o
RegCM3/Grell coloca uma banda de precipitação mais ao sul do campo observado, o que pode indicar a presença de sistemas sinóticos. Desta maneira, o modelo tenta representar aspectos físicos da dinâmica extratropical.

\subsubsection{Viés}

Nesta sessão será mostrado o viés que representa a diferença entre a precipitação observada e a simulada pelo RegCM3/Grel para DJF e MAM para a média dos cinco membros de previsão (ENSEMBLE) (Figura 6). O viés entre -100 e 100 $\mathrm{mm}$, indica que o modelo foi capaz de representar a banda de precipitação associada à ocorrência de SF ou de ZCAS localizada no setor centro-sul da região de estudo, durante o verão (Figura 6). Isto sugere que o modelo está, relativamente, mais ajustado para simular os processos termodinâmicos de latitudes extratropicais. Todavia, os valores de viés negativos (Figura 6), podem estar relacionados a erros sistemáticos de subestimativa, mostrando que o modelo esta colocando seca onde deveria colocar chuva. Ou seja, o modelo está executando processos físicos opostos ao que foi proposto. No entanto, espacialmente, o viés negativo indica que o modelo tenta reproduzir o padrão chuvoso de verão, apesar do efeito reverso. Ainda para o viés negativo, para a 


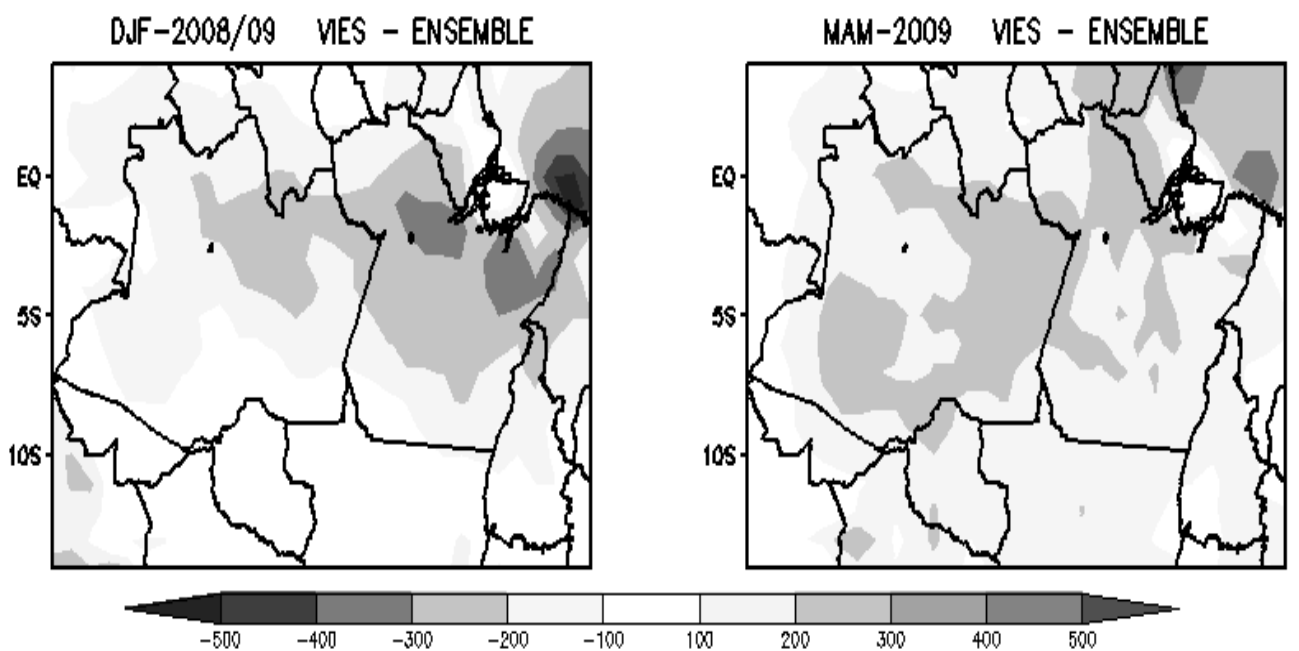

Figura 6 - Viés da precipitação (mm) sazonal para DJF-2008/09 (painel á esquerda) e MAM-2009 (painel á direita) sobre a Amazônia para a média dos 5 membros (ENSEMBLE).

estação de outono, se observa que o modelo tentar reproduzir a distribuição da chuva de modo muito preliminar em grande parte do domínio mostrado. Estes desvios podem estar representando a falta de ajuste do esquema Grell às características físicas da região Amazônica, uma vez que, nas áreas da atuação dos SF e da ZCAS, se nota os melhores resultados. Portanto, isto também revela, que talvez a baixa resolução usada, não seja o fator mais decisivo na reprodução da sazonalidade da precipitação durante o verão e o outono na Amazônia.

\section{CONCLUSÃO}

Atualmente, há poucos estudos na área de modelagem climática para a região Amazônica, principalmente, que tenham como objetivo estudar quanto o Atlântico Equatorial contribui para o padrão sazonal da estação chuvosa. Portanto, o presente estudo traz uma visão diferenciada dos demais estudos realizados, contribuindo assim, através da modelagem climática, para futuros estudos de variabilidade pluviométrica da região Amazônica. Desta maneira, os principais resultados encontrados neste trabalho foram:

- Os aspectos dinâmicos, em superfície, foram reproduzidos pelo modelo RegCM3/Grell de modo qualitativo sobre parte do oceano atlântico equatorial e região norte e nordeste do Brasil, durante o verão e outono;

- Sobre o continente, o escoamento em altos níveis simulado foi próximo ao observado, exceto para a localização do centro da Alta da Bolívia, a discordância na simulação do cavado do Nordeste e o deslocamento das altas pressões, durante o outono;

- A precipitação na região Amazônica durante o verão, especialmente sobre o centro-sul da região, foi reproduzida de modo análogo com a observação, devido à atuação da ZCAS ou SF e linhas de instabilidades;

- No outono, os processos convectivos associados à precipitação da ZCIT, foram pouco notados pelo RegCM3/Grell, em contrapartida os efeitos locais, a precipitação relacionada às linhas de instabilidades e os aglomerados foram reproduzidos;

- No verão, os valores negativos de viés indicam, que o RegCM3/Grell apresenta um efeito reverso. O mesmo tenta, mesmo que espacialmente, capitar a distribuição das chuvas na estação;

- O esquema Grell, se revela bem adequado nas áreas em que os movimentos convectivos sejam determinantes na caracterização do tempo e clima;

- Observou-se que o esquema Grell e a resolução da grade, podem ter sido fatores limitantes para a obtenção de melhor desempenho do modelo RegCM3. No entanto, para as áreas de influência do SF e eventos de ZCAS não se notaram erros tão grosseiros nas representações.

Todavia, a partir dos resultados obtidos se pretende fazer, para estudos futuros, primeiramente, uma configuração das simulações mais adequadas para a região. Fazer a utilização de esquemas de convecção que mais se aproximem das características convectivas, de modelos de superfície que melhor descrevam as propriedades físicas e biológicas da região, empregar alta resolução nas suas rodadas e criar todo um aparado que gere melhores resultados para utilizar, o mesmo, de modo operacional nos centros de previsão da região norte.

\section{REFERÊNCIAS BIBLIOGRÁFICAS}

ARKIN, P. A.; MEISNER, B. The relationship between largescale convective rainfall and cold cloud over the Western 
Hemisphere during 1982-1984. Monthly Weather Review, v.115, p. 51-74. 1987.

BERGANT, K.; BELDA, M.; HALENKA, T. Systematic errors in the simulation of European climate (1961-2000) with RegCM3 driven by NCEP/NCAR reanalysis. International Journal of Climatology, v. 27, p. 455-472, 2007.

BUIZZA, R.; HOLLINGSWORTH, A.; LALAURETTE, F.; GUELLI A. Probabilistic Predictions of Precipitation Using the ECMWF Ensemble Prediction System. Weather and Forecasting. v. 14, p. 168-189, 1999.

CARVALHO, L. M. V.; JONES, C.; SILVA DIAS, M. A. F. Intraseasonal large-scale circulations and mesoscale convective activity in Tropical South America during the TRMM-LBA campaign. Journal of Geophysical Research., v. 29, n. 10.102/2001JD000745. 2002.

COHEN, J. C. P.; SILVADIAS, M.A.; NOBRE, C. Environmental Conditions Associated with Amazonian Squall Lines: A Case Study. Monthly Weather Review, v.123, n.11, p.31293143, 1995.

CORREIA, F. W. S; MANZI, A. O.; CÂNDIDO, L. A.; SANTOS, R. M. N.; PAULIQUEVIS, T. Balanço de umidade na Amazônia e sua sensibilidade às mudanças na cobertura vegetal. Ciência e Cultura, São Paulo, v. 59, n.3. jul./set. 2007.

CUADRA, S. V.; ROCHA, R. P. Simulação numérica do clima de verão sobre o Brasil e sua variabilidade. Revista Brasileira de Meteorologia, v. 21, n. 2, p. 271-282, 2006. De SOUZA, E. B. KAYANO, M. T.; TOTA J.; PEZZI L.; FISCH, G.; NOBRE, C. On the influences of the El Niño, La Niña and Atlantic dipole pattern on the Amazonian rainfall during 1960-1998. Acta Amazonica, v. 30, n. 2, p. 305-318, 2000.

De SOUZA, E. B.; AMBRIZZI, T. ENSO impacts on the South American rainfall during 1980s: Hadley and Walker circulation. Atmósfera, v. 15, p. 105-120, 2002.

De SOUZA, E. B.; AMBRIZZI, T. Pentad precipitation climatology over Brazil and the associated atmospheric mechanisms. Climanálise, v. 1, p. 1-20, 2003. [Available online at http:// www.cptec.inpe.br/products/climanalise/ artigos/artcien2. shtml].

De SOUZA, E. B.; KAYANO, M. T.; AMBRIZZI, T. The regional precipitation over the eastern Amazon/northeast Brazil modulated by tropical Pacific and Atlantic SST anomalies on weekly timescale. Revista Brasileira de Meteorologia, v. 19, n. 2, p. 113-122, 2004.

De SOUZA, E. B.; ROCHA, E. J. P. Diurnal variations of rainfall in Bragança-PA (eastern Amazon) during rainy season: mean characteristics and extreme events. Revista Brasileira de Meteorologia, v. 21, n. 3, p. 142-152, 2006.

De SOUZA, E. B.; AMBRIZZI, T. Modulation of the Intraseasonal Rainfall over Tropical Brazil by the Madden-
Julian Oscillation. International Journal of Climatology, DOI: 10.1002/joc.1331. 2006.

De SOUZA, E. B.; LOPES, M. N. G.; ROCHA, E. J. P.; SOUZA, J. R. S.; CUNHA, A. C.; SILVA, R. R.; FERREIRA, D. B. S.; SANTOS, D. M.; CARMO, A. M. C.; SOUSA, J. R. A.; GUIMARÃES, P. L.; MOTA, M. A. S.; MAKINO, M.; SENNA, R. C.; SOUSA, A. M. L.; MORA, G. V.; KUHN, P. A. F.; SOUZA, P. F. S.; VITORINO, M. I. Precipitação sazonal sobre Amazônia Oriental no período chuvoso: Observações e simulações regionais com o RegCM3. Revista Brasileira de Meteorologia, v.24, n.2, 111-124, 2009.

DICKINSON, R. E. ERRICO R. M.; GIORGI F.; BATES G. T. A regional climate model for the western United States. Climate Change, p.383-422. v. 15. 1989.

DICKINSON, R. E.; HENDERSON-SELLERS, A.; KENNEDY, P. J. Biosphere-atmosphere transfer scheme (BATS) version $1 \mathrm{E}$ as coupled to the NCAR Community Climate Model. Boulder, Colorado. NCAR Technical Report, NCAR/TN387, 72f. 1993.

FIGUEROA, S. N.; NOBRE, C. A. Precipitation distribution over central and western tropical South America. Climanálise, v. 5, p. 36-45, 1990.

GIORGI, F.; BATES, G. T. The climatological skill of a regional climate model over complex terrain. Monthly Weather Review, v. 117, p.2325-2347. 1989.

GIORGI, F.; MARINUCCI, M. R.; BATES, G. T. Development of a Second-Generation Regional Climate Model (RegCM2). Part I: Boundary-layer and radiative transfer processes. Monthly Weather Review, v. 121, p.2794-2813. 1993a.

GIORGI, F.; MARINUCCI M. R.; BATES G. T. Development of a second-generation regional climate model (RegCM2). Part II: convective processes and assimilation of lateral boundary conditions. Monthly Weather Review, v. 121, p. 2814-2832, 1993b.

GIORGI, F.; MEARNS, L. O. Introduction to special section: regional climate modeling revisited. Journal of Geophysical Research, v. 104, p.6335-6352. 1999.

GRELL, G. A. Prognostic evaluation of assumptions used by cumulus parameterizations. Monthly Weather Review, v.121, p.764-787. 1993.

HEWITSON, B. C.; CRANE, R. G.; Climate downscaling: techiques and application. Climate Research, v. 7. p. 8595. 1996.

KALNAY, E.; KANAMITSU, M.; KISTLER, R.; COLLINS, W.; DEAVEN, D.; GANDIN, L.; IREDELL, M.; SAHA, S.; WHITE, G.; WOOLLEN, J.; ZHU, Y.; LEETMA, A.; REYNOLDS, B.; CHELLIAH, M.; EBISUZAKI, W.; HIGGINS, W.; JANOWIAK, J.; MO, K. C.; ROPELEWSKI, C.; WANG, J.; JENNE; ROY; JOSEPH; DENNIS; NCEP/ 
NCAR 40-year Reanalysis project. Bulletin of the American Meteorological Society, v. 77, p. 437-471, 1996. KIEHL, J. T.; HACK, J. J.; BONAN, G. R.; ROVILLE, B. A.; BRICGLCB, B. P.; WILLIAMSON, D. L.; RASCH P. J. The National Center for Atmospheric Research Community Climate Model: CCM3. Journal of Climate, v. 11, p. 1131- 1149, 1998.

KOUSKY, V. E.; CAVALCANTI, I. F. Precipitation and atmospheric circulation anomaly patterns in the South American sector, Revista Brasileira de Meteorologia, v.3, 199-206, 1988.

LEUNG, L. R.; QIAN Y.; BIAN, X. D.; WASHINGTON, W. M.; HAN, J. G.; ROADS, J. O. Mid-Century Ensemble Regional Climate Change Scenarios for the Western United States. Climate Change. v. 62. P. 75-113. 2004.

LIANG, X.; PAN, J.; ZHU, J.; KUNKEL, K. E.; WANG, J. X. L.; DAI, A. Regional climate model downscaling of the U. S. summer climate and future change. Journal of Geophysical Research, v. 111. 2006

LOPES, M. N. G. Aspectos regionais da variabilidade de precipitação no estado do Pará: estudo observacional e modelagem climática em alta resolução. 2009. 117 f. Dissertação ( Mestrado em Ciências Ambientais) Universidade Federal do Pará, Instituto de Geociências, Museu Paraense Emílio Goeldi, Embrapa Amazônia Oriental, Programa de Pós-Graduação em Ciências Ambientais, Belém, 2009.

MACHADO, L. A. T.; LAURENT, H.; DESSAY, N.; MIRANDA, I. Seasonal and diurnal variability of convection over the Amazonia - A comparison of different vegetation types and large scale forcing. Theoretical and Applied Climatology, 78, 61-77, 2004.

MARENGO, J. A.; HASTENRATH, S. Case studies of climatic events in Amazon basin. Journal of Climate, v. 6, n.4, p.617-627. 1993.

MENÉNDEZ, C. G.; SAULO, A. C.; LI, Z. X. Simulation of South American wintertime climate with a nesting system. Climate Dynamics, v. 17. n. 2-3 p. 219-231. 2001.

MISRA, V.; DIRMEYER, A. P.; KIRTMAN, B. P. Dynamic downscaling of seasonal simulation over South America. Journal of Climate, v.16, p. 103-117, 2003.

MOLION, L. C. B. Climatologia dinâmica da Região Amazônica: mecanismos de precipitação. Revista Brasileira de Meteorologia, 2, 107-117. 1987.

MOLTENI, F.; BUIZZA, R.; PALMER, T. N.; PETROLIAGIS, T. The ECMWF Ensemble Prediction System: Methodology and Validation. Quarterly Journal of the Royal Meteorological Society, v. 122, p. 73-119, 1996.

MOURA, A. D.; SHUKLA, J. On the dynamics of the droughts in Northeast Brazil: observations, theory and numerical experiments with a general circulation model. Journal of the Atmospheric Science, v. 38, p. 2653-2673, 1981.

NICOLINI, M.; SALIO, P.; KATZFEY, J. J.; McGREGOR, J. L.; SAULO, A. C. January and July regional climate simulation over South American. Journal of Geophysical Research, v. 107 . n. D22. 2002.

NOBRE, P.; MOURA, A.D.; SUN, L. Dynamical downscaling of seasonal climate prediction over Nordeste Brazil with ECHAM3 and NCEP's regional spectral model at IRI. Bulletin of the American Meteorological Society, v. 82, p. 2787-2796, 2001

PAL, J. S.; SMALL E. E.; ELTAHIR E. A. B. Simulation of regional-scale water and energy budgets: Representation of subgrid cloud and precipitation processes within RegCM. Journal of Geophysical Research. v. 105. p. 29-45. 2000. PAL, J. S.; GIORGI, F.; XUNQIANG, B.; ELGUINDI, N.; SOLMON, F.; GAO, X.; RAUSCHER, S. A.; FRANCISCO, R.; ZAKEY, A.; WINTER, J.; ASHFAQ, M.; SYED, F. S.; BELL, J. L.; DIFFENBAUGH, N. S.; KARMACHARYA, J.; KONARÉ, A.; MARTINEZ, D.; ROCHA, R. P. SLOAN, L. C.; STEINER, A.. The ICTP RegCM3 and RegCNET: regional climate modeling for the developing World. Bulletin of the American Meteorological Society, v. 1, p. 1395-1409, 2007.

QUIAN, J. H.; SETH, A.; ZEBIAK, S. Reinitialized versus continuous imulations for regional climate downscaling. Monthly Weather Review, v. 131. n. 11. p. 2857-2874. 2003.

REYNOLDS, R.W.; RAYNER, N. A.; SMITH, T. M.; STOKES, D. C.; WANG, W. An improved in situ and satellite SST analysis for climate. Journal of Climate, v. 15, p. 1609 $1625,2002$.

ROPELEWSKI, C. F., HALPERT, M. S. Global and Regional Scale Precipitation Patterns Associated with the El-Niño Southern Oscillation. Monthly Weather Review,. 115, 1606-1626. 1987

SEGELE, Z. T.; LESLIE, L. M.; LAMB, P. J. Evaluation and adaptation of a regional climate model for the Horn of Africa: rainfall climatology and interannual variability. International Journal of Climatology, 29: 47-65 (2009) Published online 18 April 2008 in Wiley InterScience. DOI: 10.1002/joc. 1681

SETH, A.; ROJAS, M. Simulation and sensitivity in a nested modeling system for South America. Part I: reanalyses boundary forcing. Journal of Climate, v. 16, p. 2437-2453, 2003.

SILVA, V. B.; KOUSKY V. E.; SHI W.; HIGGINS, R. W. An improved gridded historical daily precipitation analysis for Brazil. Journal of Hydrometeorology, v. 8, p. 847-861, 2007. 
SLINGO, J. M.: A gcm parameterization for the shortwave radiative properties of water clouds. Journal of the Atmospheric Science, 46, 1419-1427. 1989.

STEINER, A. L.; PAL J. S.; RAUSCHER S. A.; BELL J. L.; DIFFENBAUGH N. S.; BOONE A.;SLOAN L. C.; GIORGI F. Land surface coupling in regional climate simulations of the West African monsoon. Climate Dynamic, 33, 869892,2009 .

SUN, L.; MONCUNILL, D. F.; LI, H.; MOURA, A. D.; FILHO, F. A. S.; ZEBIAK, S. E. An operational dynamical downscaling prediction system from Nordeste Brazil and the 2002-04 real-time forecast evaluation. Journal of Climate, v. 19, p. 1990-2007, 2006.
VITORINO, M. I.; SILVA DIAS, P. L.; FERREIRA, N. J. Observational study of the seasonality of the submonthly and intraseasonal signal over the tropics. Meteorology and Atmospheric Physics, 93, 17-35. 2006.

WILKS, D. S.; Multisite downscaling of daily precipitation with a stochastic weather generator. Climate Research. v. 11. p. 125-136. 1999.

WILKS, D. S.; Statistical methods in the atmospheric sciences. 2. Ed. San Diego: Elsevier, 627p. 2006. 